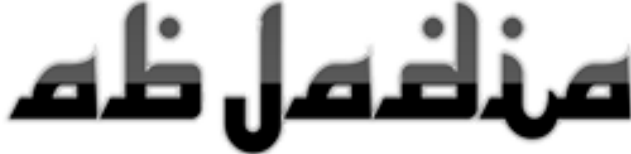 \\ International Journal of Education}

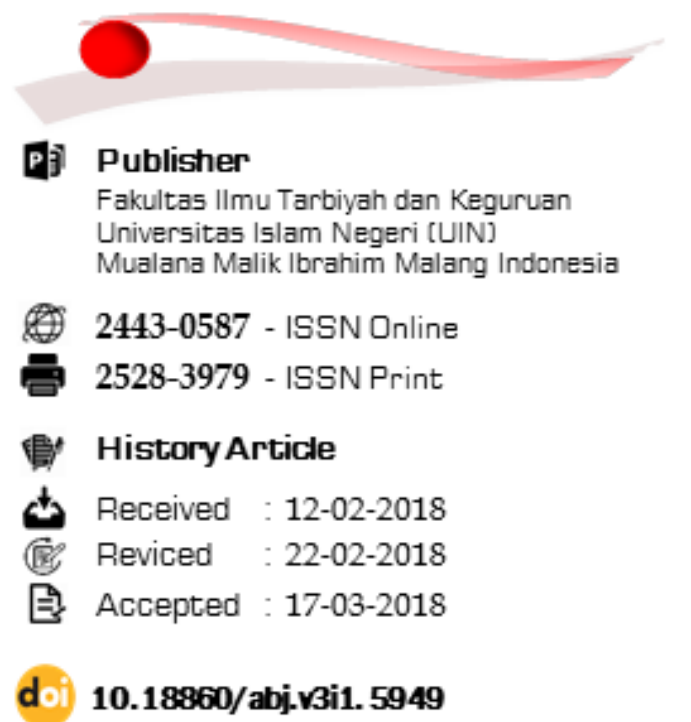

A http://ejournal.uin-malang.ac.id/index.php/ abjadia/article/view/5949

(2) Dwi Maihidin Pahlepi
(2) Afreni Hamidah
(2) Mia Aina
(2) Indonesia
(3) Universitas Jambi
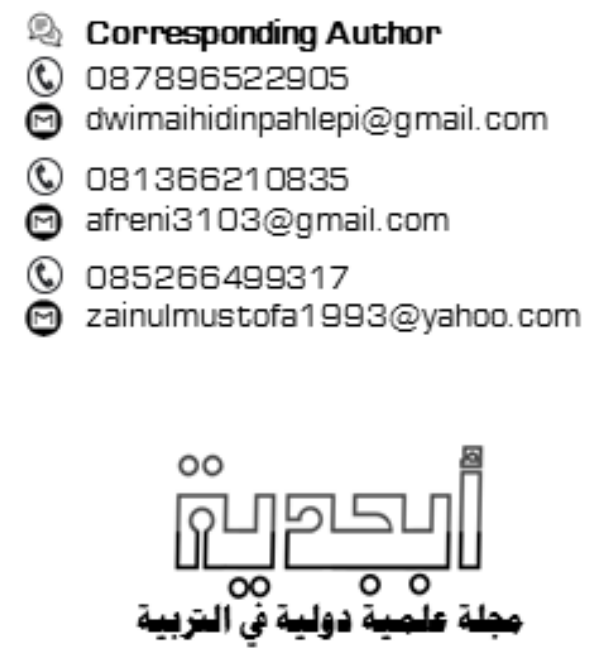

Dwi Maihidin Pahlepi, Afreni Hamidah, Mia Aina Indonesia

تطوير معجم بيولوجيا على أسـاس أندرويد في المواد اللافقارية لطلاب مدرسة الثانوية للصف العاشر ببرنامج الرياضية

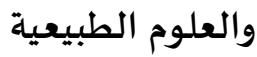

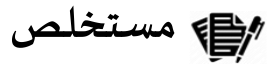

اللافقاريات هي واحدة من المواد التي يجب دراستها في البيولوجي

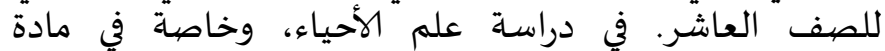

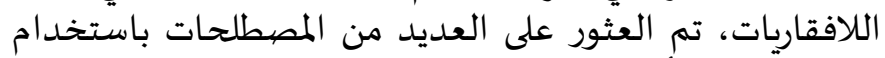

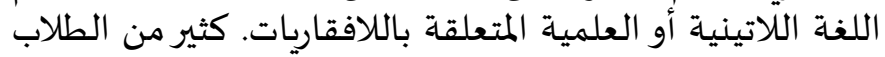

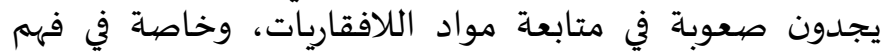

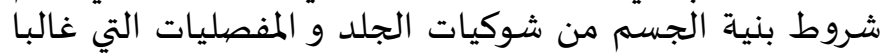

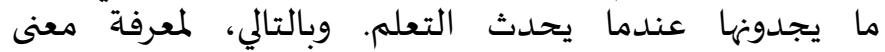

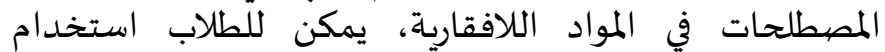

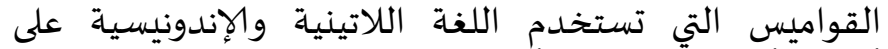

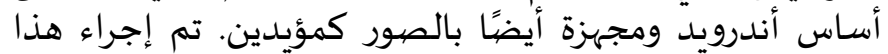

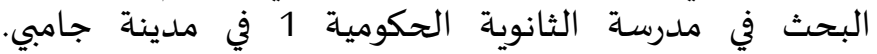

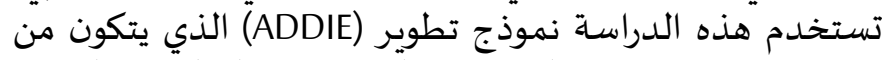

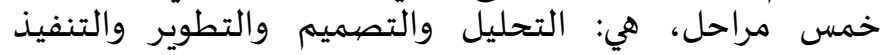

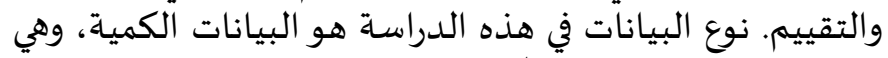

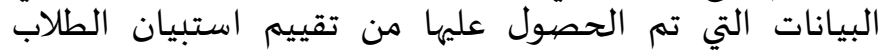

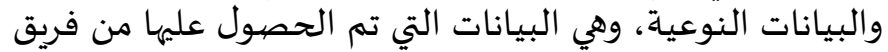

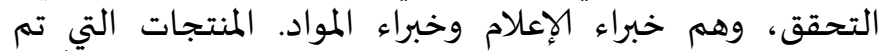

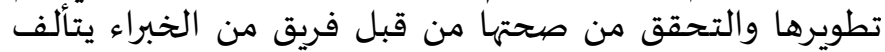

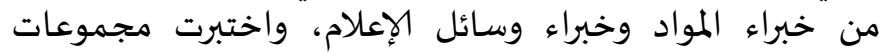

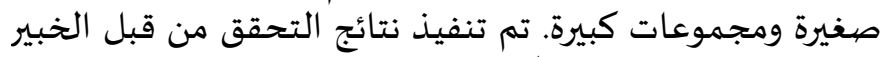

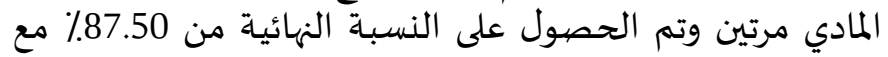

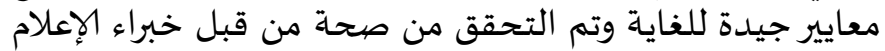

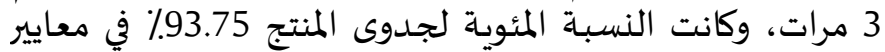

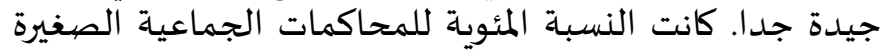

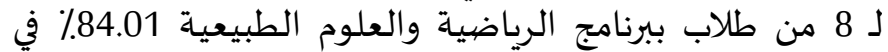

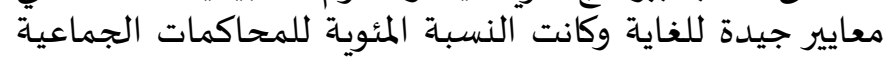

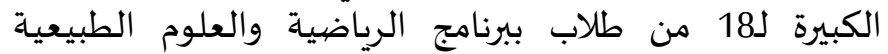

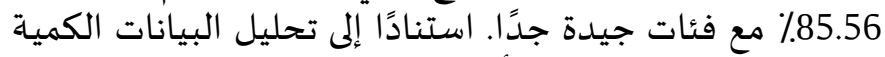

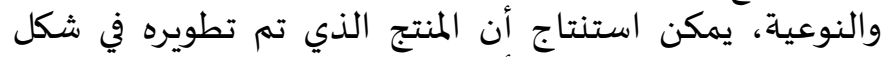

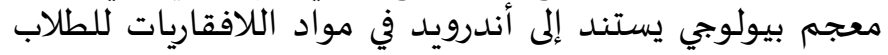

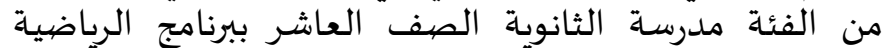
والعلوم الطبيعية من الممكن استخدامدة في عملية التعلم.

$$
\text { هeمجم الوسائط للبيولوجيا، أندرويد، اللافقاريات }
$$

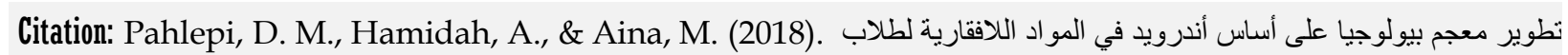

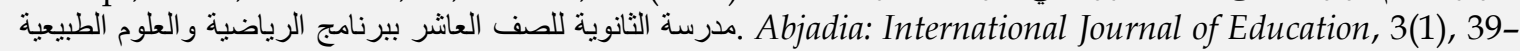
50. 


\section{嘼”" Abstract}

Invertebrates is one of the compulsory materials in Biology lesson of class X. In studying biology, there are lots of terms using Latin or scientific language related to invertebrates. Many students have difficulty in learning Invertebrates material, especially in understanding the terminology section of the body structure of Echinoderms and Arthropods. Thus, to determine the meaning of the terms in these invertebrates materials students can use a dictionary that provides Latin and Indonesian languages based on Android and is also equipped with supporting images. This research was conducted at Senior High School 1 Jambi. This study aims to make learning media in the form of dictionary-based android biology in invertebrate material and test their feasibility with a small group trial. This study using ADDIE development model consisting of five stages: analysing, designing, developing, implementing and evaluating. The type of data in this study is a quantitative data which is obtained from the assessment of student questionnaire and qualitative data that is obtained from the validation team who are media and subject matter experts. The products have been developed and validated by a team of experts consisting of subject matter experts and media experts, tested to small groups and large groups. The results of the validation by subject matter experts which held 2 times gain the final percentage $87.50 \%$ in the criteria very well and the validation by media experts conducted 3 times, produces a percentage of $93.75 \%$ and the product eligibility criteria is very well. The percentage of small group trial to 8 students MIPA is $84.01 \%$ in the criteria very well and the percentage of large groups of trials to 18 students MIPA is $85.56 \%$ in the excellent category. Based on the quantitative and qualitative data analysis, it can be concluded that the products developed in the form of the Dictionary of Biology Android based on Material of Invertebrates for High School Students of X Graders in Science Class is eligible to be implemented in the learning process.

Media Dictionary of Biology, Android, Invertebrates

\section{(C) مقدمة}

$$
\begin{aligned}
& \text { المنهاج والتعلم هما شيئان لا يمكن فصلهما. المناهج الدراسية هي أداة لتحقيق الأهداف } \\
& \text { التعليمية ، بالإضافة إلى مبادئ توجيهية حول تطبيق التعليم ، يجب إصلاح المناهج لأنه لا يوجد منهج } \\
& \text { واحد يناسب كل الوقت ، يجب على المناهج أن تتكيف مع تطور العصر الذي يميل دائمًا إلى التغيير. } \\
& \text { وفقا لتوتوك في سيرامبينا (2016: 3) بدءا من العام الدراسي الجديد 2017/2016، ستقوم وزارة التعليم } \\
& \text { والثقافة (وزارة التربية) رسميا بسن المناهج الدراسية لعام } 2013 \text { على المستوى الوطني. النقاط الهامة في } \\
& \text { مراجعة المنهاج لعام r| ب هي: .1 تبسيط جوانب تقييم الطلاب من قبل المعلم، .2 عمالية تفكير }
\end{aligned}
$$


الطلاب غير هحدودة. .3 إن نظرية 5) التذكر ، الفهم، التطبيق، التحليل، والقراءة)، ليست مجرد نظرية. ومع ذلك ، يُطلب من المعلم أن يتقدم بطلب حقيقي في التعلم، 4. لا يتم تغيير بنية المواد الدراسية وطول الدراسة في المدرسة. تعتبر اللافقاريات من المواد التي يجب دراستها في موضوعات علم الأحياء من الفئة 10. في دراسة

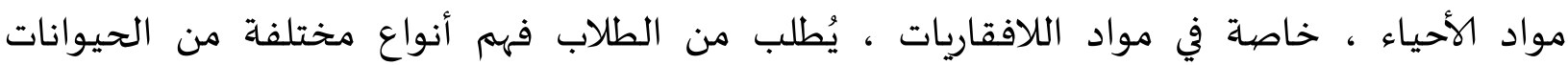

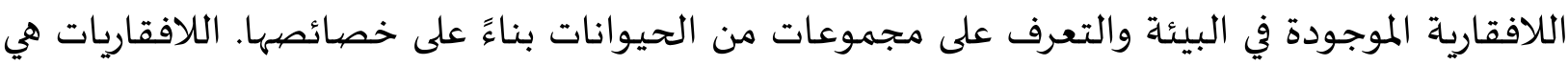

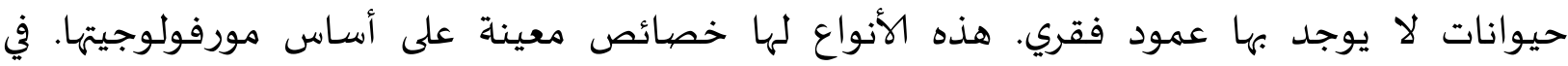
مورفولوجيا وجدت العديد من المصطلحات الهامة فيما يتعلق بخصائص اللافقاريات. لفهم هذه المصطلحات من الضروري استخدام معجم ، لكن معجم الأحياء لا يزال عامًا. استنادًا إلى نتائج الملاحظات في العديد من المدارس الثانوية العامة في مدينة جامبي ، والتي قام يها الباحثون ، فإن الطلاب لا يجلبون معجما بيولوجيًا إلى المدرسة لأن المعجم كبير وثقيل ، بالإضافة إلى ذلك لا يفهم الطلاب المصطلحات في مادة اللافقاريات. في الأنشطة التعليمية الصفية ، لا يحب الطلاب البحث عن الأسماء العلمية في المواد اللافقارية لأنه وفقًا للطلاب ، فإن معجم الأحياء ليس مثيرًا للاهتمام ومملًا ، لأن قواميس الأحياء تقدم عمومًا أسماء علمية مع تفسيرات في شكل نص. سيكون النص وفقًا للطلاب أكثر إثارة إذا ظهرت الصور. وفقًا لماير (2001: 100) باستخدام الصيور ، يمكننا رسم المادة في شكل أكثر غريزة وأقرب إلى تجربتنا الحسية. في السنوات القليلة الماضية ، أصبحت الهواتف المحمولة بنظام التشغيل أندرويد هي أجهزة

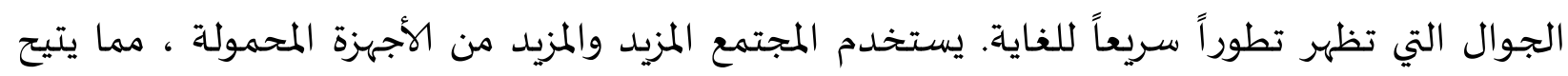
فرصًا لاستخدام الأجهزة التكنولوجية للتحرك في عالم التعليم. وفقًا لسلبنو (2014: 7)، نظام أندرويد هو نظام تشغيل قائم على نظام التشغيل لينكس مفتوح المصدر (مفتوح المصدر) ومُصمم للأجهزة الخلوية التي تعمل باللمس مثل الهواتف الذكية وأجهزة الكمبيوتر اللوحية. تم تطوير أندرويد بواسطة أندرويد، شركة، بدعم مالي من جوجل تم شراؤه لاحقًا في عام 2005. تم إصدار أندرويد رسميًا في عام 2007، إلى جانب إنشاء تحالف فتح تحالف الهاتف.

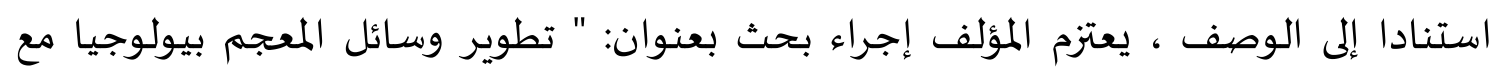
صورها على أساس أندرويد في المواد اللافقارية للطلاب مدرسة الثانوية الصف العاشر ببرنامج الرياضية

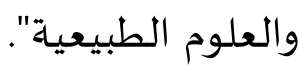




\section{[Q] منهج}

إن نوع البحث الذي يتماشى مع المشاكل في هذه الدراسة هو البحث التنموي. كما أوضح أسيم

(2001: 1) ، فإن هذا التطوير البحثي في التعلم هو بحث يستخدم لتطوير المنتجات والتحقق منها في

عملية التعلم.

وفقا لبيريبادي (2009: 125)، يعد ADDIE نموذج تصميم نظام تعليمي يوضح المراحل الأسـاسية

لتصيميم نظام التعلم البسيط والسهل التعلم.

بناءً على نموذج تطوير ADDIE، فيما يلي خطوات نموذج ADDIE.

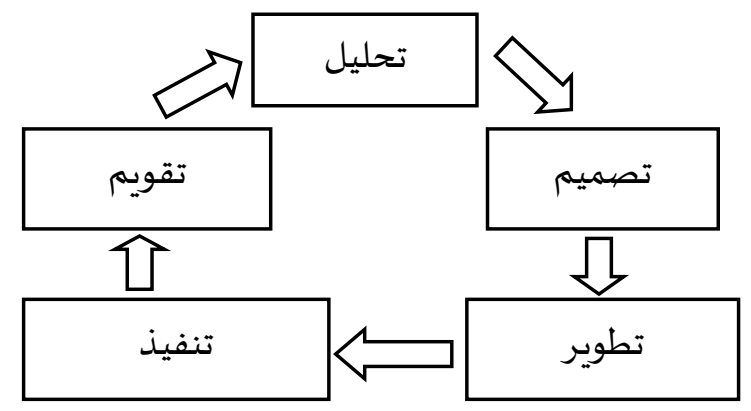

صورة 3.1: عملية تطوير نموذج ADDIE (لي و أوين، 2004: 93)

تجربة المنتج

في تصميم المنتج التجريبي ، هناك تطورات التصيميم في هذه الدراسـة بما في ذلك:

1. تصميم المنتج في شكل معجم الوسائط البيولوجية على أسـاس تصميم أندرويد في مواد الالتحام

شوكيات الجلد دان المفصليات.

2. التحقق من صحة من قبل فريق المدقق على منتجات الوسائط معجم الوسائط المستندة إلى

أندرويد في شكل أفك. يتم التحقق من الصحة قبل اختبار المنتج على المستخدم.

3. تتم مراجعة المنتج الأولي بعد أن يكون فريق أداة التحقق هو المدقق المادي وتحقق أداة التحقق

$$
\text { تجربة الموضوعات صحة المنتج الذي يتم تطويره }
$$

أجريت الدراسة على طلاب من مدرسة الثانوية الصف العاشر ببرنامج الرياضية والعلوم

الطبيعية جامبي. كان الأشخاص الذين خضعوا للدراسة في هذه الدراسة عبارة عن مجموعات صغيرة أو

موضوعات تجريبية محدودة (عددهم 8 أشخاص) واستمروا في تجربة مجموعات كبيرة (مجموعها 18 
شخصًا). وفقا لأريكنتو (2013: 254) أجري موضوعات التجارب الجماعية الصغيرة في 4-14 المجيبين والمجموعات الكبيرة بين 15-50 المجيبين. الطلاب المختارون هم الطلاب الذين لديهم جهاز محمول يعمل بنظام أندرويد. ثم قام المستجيبون بتعبئة استبيان تقييم على وسـائل الإعلام التي تم استخدامها. أنواع البيانات

يتم الحصول على هذه البيانات من نتائج التحقق من صحة من قبل فريق مدقق وسائل الإعلام والمواد المتعلقة بجدوى وسائل الإعلام المتقدمة واستجابات الطلاب عند اختبار المنتج باستخدام مقياس ليكرت. البيانات الكمية هي البيانات في شكل أرقام ، لأنه وفقا ل رضوان (2013: 5) أن البيانات النوعية هي البيانات المتعلقة بتصنيف ، والخصائص في شكل أسئلة أو في شكل كلمات. أدوات جمع البيانات في جمع البيانات في هذا البحث تم استخدام الاستبيانات كأدوات. الاستبيان الذي تم توزيعه هو استبيان مفتوح واستبيان مغلق. يتم تقديم استبيانات مفتوحة للطلاب أثناء تجارب المنتج، بينما يتم استخدام الاستبيانات المغلقة أثناء عملية التحقق من الصحة بواسطة فريق الخبراء. تحليل البيانات

البيانات التي تم الحصول عليها في هذه الدراسـة هي البيانات الكمية والنوعية. يتم تحليل البيانات عن طريق حساب النتيجة. إجابات المجيبين التي تم جمعها من خلال الاستبيانات التي أعطيت الدرجات في كل خيار للإجابة ، قبل تحليل الاستبيان يتم جدولتها في شكل جدولي بحيث يتم الحصول على عدد الدرجات التي تم الحصول عليها من إجابات الطلاب. تم تحليل البيانات عن طريق حساب متوسط النسبة المئوية للخطوات الشاملة التي تم الحصول عليها من الاستبيان باستخدام المعادلة التالية:

$$
\begin{aligned}
\%=\frac{\mathrm{F}}{\mathrm{N}} \times 100 \% \\
\text { ملاحظات: }
\end{aligned}
$$

\% = النسبة المئوية المتغيرة الفرعية = عدد القيم لكل متغير فرعي = F = مجموع النقاط القصوى 


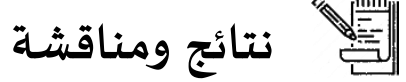 \\ تحليل البيانات من خبير التحقق من المواد}

استناداً إلى نتائج التحقق من صحة المرحلة الأولى من المواد التي حصلت على درجة تقييم من المواد

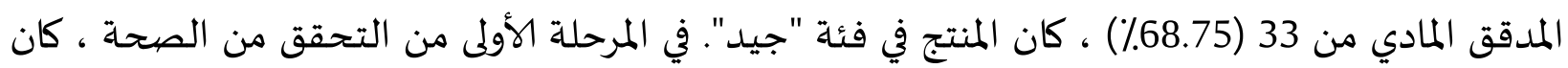
هناك العديد من الاقتراحات للتحسين من قبل خبراء المواد ، وهي تصحيح تفسيرات المفردات التي كانت

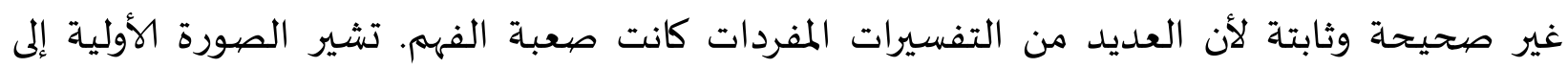
الهيكل العام للمفردات على جسم حيوان اللافقاريات محل صورة أكثر تحديدًا بحيث تكون الصورة أكثر

وضوحًا للرؤية. تم تصحيح بنية جملة تفسير المفردات التي كانت فوضوي في البدان البداية وأكثر تبسيطًا.

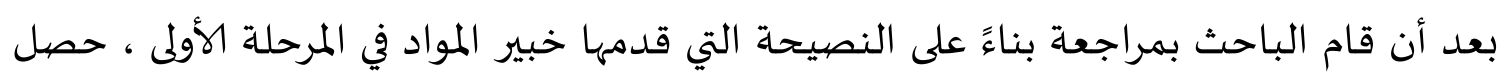

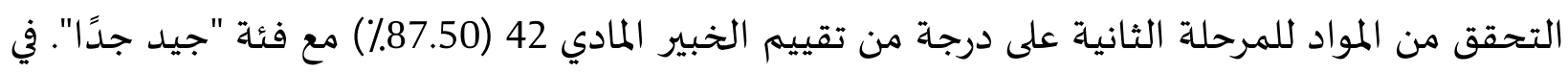

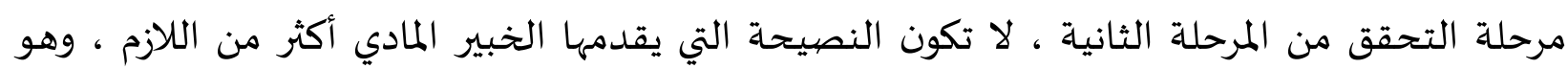
اختيار واحد فقط من نفس تفسيرات المفردات للمفردات مثل غدة السم أو مفردات السموم. في المرحلة

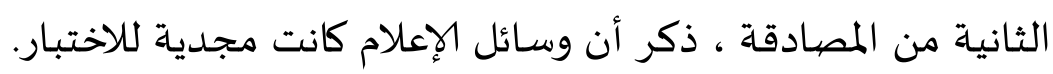

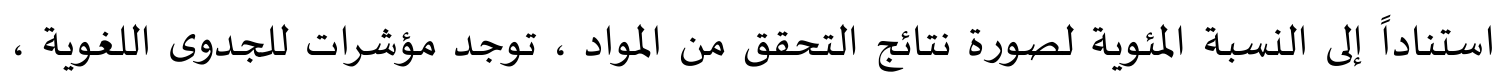
حيث زادت المرحلة الأولى (58.33\%) بنسبة 25\% بحيث تكون في المرحلة الثانية (83.33\%). على مرحلة مؤشر جدوى المحتوى الأول (78.57 \%) بنسبة 10.72 \% بحيث في المرحلة الثانية (89.29 \%). بالإضافة إلى الى مؤشرات الدقة والموثوقية في المرحلة الأولى مع المرحلة الثانية ، لا توجد زيادة لأن القيمة المعطاة في هذه المهاد الفئة جيدة جدًا. من بين المؤشرات الثلاثة التي شهدت زيادة كبيرة ، أي مؤشر الجدوى اللغوية.

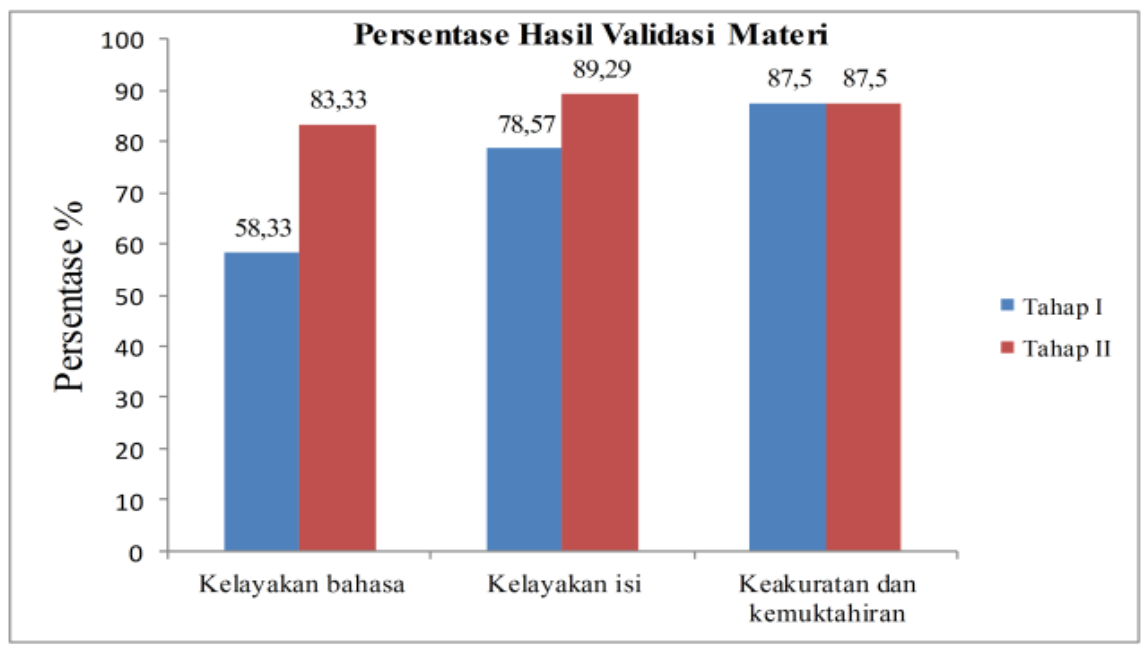

صورة 4.1 النسبة المئوية لنتائج التحقق من المواد 
تحليل البيانات من التحقق من صحة وسـائل الإعلام

واستناداً إلى نتائج التحقق من صحة وسائل الإعلام للمرحلة الأولى ، كانت النتيجة التي تم الحصول عليها من مدقق الوسائط 29 (60.41\%) ، وكان المنتج في فئة "جيد". في المرحلة الأولى من التحقق من الصحة ، هناك العديد من الاقتراحات للتحسين من قبل خبراء الإعلام ، وبالتحديد على أزرار كفاءة الرئسية و كفاءة الأسـاسية في القائمة الرئيسية ، يتم استبدال معجم البيولوجيا القائم على أندرويد بزرّ "حول" للتطبيق نظرًا لأن المنتج الذي تم تطويره هو معجم ليس وسيطًا للتعلم يفسر موضوع الدراسة. يجب تكبير وعرض زر صغير في الجزء السـفلي من الزر الموصى باء وتحديد موقعه في المنتصف. يُوصى باستبدال حجم الخط الذي كان بحجم 12 مسبقًا بحجم 14 أو 16 ، ويوصى باستخدام لون الخط الذي كان في الأصل مشرقًا مع خلفية زاهية باستخدام خط داكن. كان المقطع الصيوتي السـابق عبارة عن موسيقى واحدة فقط ، وقد أوصي بإضـافة أشكال موسيقية أخرى مثيرة للاهتمام. يتم اقتراح صور التراكيب في اللافقاريات التي كانت غير واضحة في السابق ولا يمكن تكبيرها بحيث يمكن تكبير الصيور دون تغيير شكل الصورة. بعد أن قام الباحث بمراجعة بناء على النصيحة التي قدمها خبراء الإعلام في المرحلة الأولى ، ثم حصلت المرحلة الثانية من التحقق من وسائل الإعلام على 33 خبيرا في الإعلام ، ثم كانت النسبة 65.75٪ مع فئة "جيد". في مرحلة التحقق من المرحلة الثانية ، لم يعد النصح الذي قدمه خبراء الإعلام أكثر من اللازم ، وبالتحديد لا تزال هناك صور غير واضحة يتم توضيحها مرة أخرى. يُقترح أن يكون لون الخط الأقل تناغمًا على الزر أكثر توافقًا مع الزر. يشير زر الرمز وشكل الزر إلى المزيد من الابتكارات لتكون أكثر

جاذبياة.

بعد أن قام الباحث بإجراء مراجعة مبنية على النصيحة التي قدمها خبراء الإعلام في المرحلة الثانياة. ثم حصلت المرحلة الثالثة من التحقق من وسائل الإعلام على درجة تصنيف من 45 من خبراء الإعلام ، ثم كانت النسبة 93.75٪ مع فئة "جيد جدًا". في مرحلة التحقق من المرحلة الثالثة لم تكن هناك اقتراحات للتحسينات المقدمة من قبل خبراء الإعلام. لذلك يقال إن وسـائل الإعلام مجدية لإنتاجها دون مراجعة.

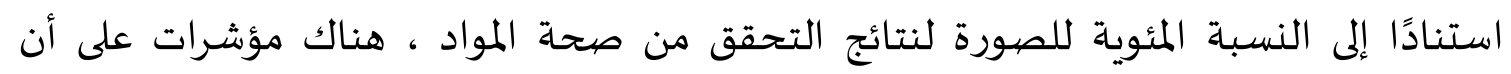
مظهر البرنامج في المرحلة الأولى (\% 57.14) قد زاد بنسبة 10.72\% بحيث أناه في المرحلة الثانية (67.86٪) ، فإن هناك زيادة بنسبة 25٪ في المرحلة الثالثة (92 ، 86٪). في مؤشر الجودة ، شهدت التقنية في المرحلة 
الأولى (65\%) زيادة بنسبة 5\% ، بحيث ارتفعت في المرحلة الثانية (70٪) ، ثم زادت مرة أخرى بنسبة 25٪ ، بحيث تكون في المرحلة الثالثة (95٪). ومن بين المؤشرين ، توجد أعلى ذسبة في مؤشرات الجودة الفنية.

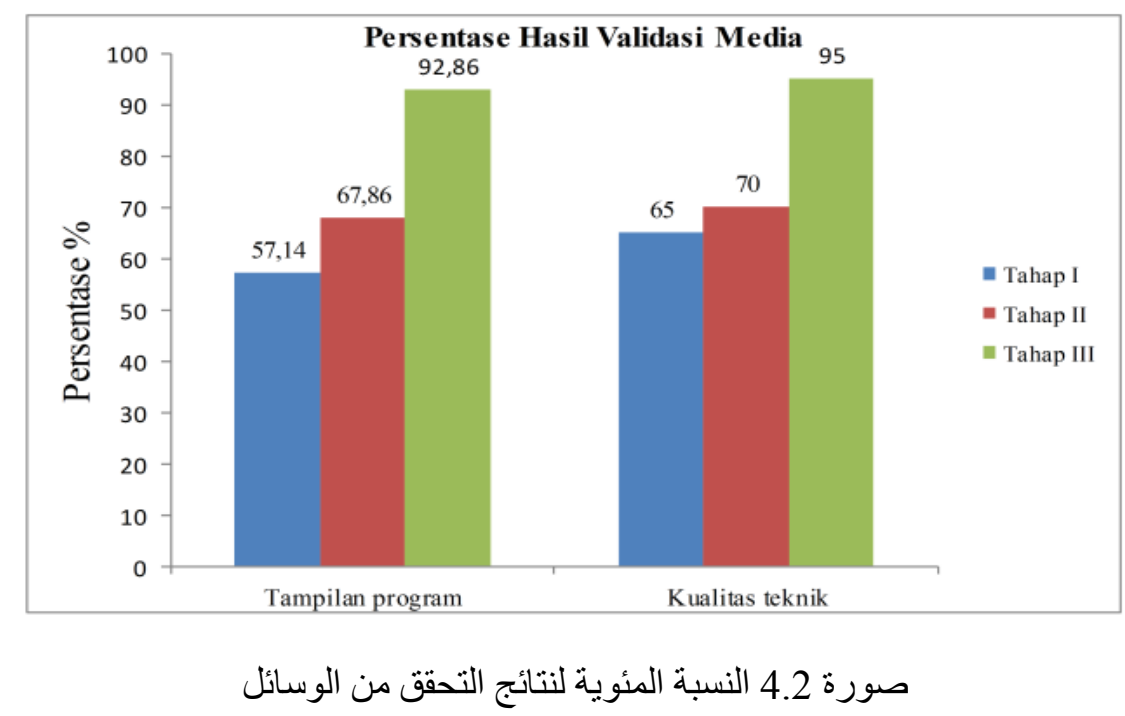

تحليل بيانات اختبار المنتج

أجريت تجارب جماعية صغيرة في مدرسة الثانوية الصف العاشر ببرنامج الرياضية والعلوم

الطبيعية مع ما مجموعاه 8 طلاب حصلوا على 475 نقطة بنسبة بلغت 84.01\% ضمن الفئة الجيدة. وبالتالي يمكن القول أن الوسائط المستندة على معجم أندرويد البيولوجي مناسبة للاستخدام في عملية التعلم. استنادًا إلى تفسير ردود الطلاب على مؤشرات عرض الوسائط ، يسجلون 82.82٪. يتم تضهين النتيجة في الفئة الممتازة ، ولكنها تحتل أقل نسبة من جميع مؤشرات تجربة المنتج. وذلك لأن مجموعات الألوان المستخدمة في المعجم أقل تنوعًا وأذواق كل طالب مختلفة الألوان. على مؤشر سهولة الاستخدام ، يتم تضمين درجة 85.42 ٪ في الفئة الممتازة ، تحتل هذه الدرجة أعلى ذسبة من جميع مؤشرات تجربة المنتج. وذلك لأن اكتمال الزر ومفتاح الجمع سهل الاستخدام. بالإضيافة إلى مؤشرات فعالية وسائل الإعلام التي حصلت على درجة 84.9٪ مشمولة في الفئة الجيدة جدًا، تحتل هذه المرتبة مركز ثاني أعلى نسبة من درجة مؤشر سهولة الاستخدام. 


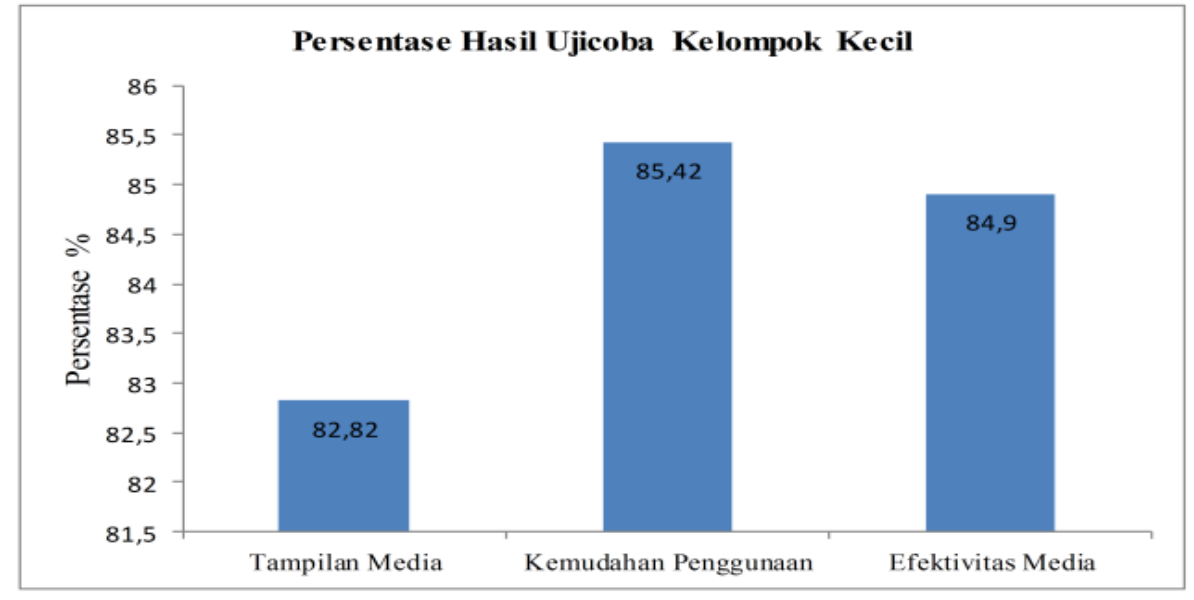

صورة 4.3 النسبة المئوية لنتائج تجارب المجمو عات الصغيرة

أجريت تجارب جماعية كبيرة في مدرسة الثانوية الصف العاشر ببرنامج الرياضية والعلوم

الطبيعية مع عدد من الطلاب بلغ عددهم 18 شخصيًا حصلوا على 1232 نقطة بنسبة 85.56٪ ضمن الفئة الجيدة. وبالتالي يمكن القول أن الوسائط المستندة إلى معجم الأحيائي في أندرويد هي مناسبة للاستخدام في عملية التعلم. يشتمل الاستبيان المستخدم في هذه التجربة الجماعية الصغيرة على (أ) عرض الوسائط ، (ب) سهولة الاستخدام (ج) فعالية وسـائل الإعلام ، (د) الكفاءة والفعالية. (ه) الفوائد. استناداً إلى تفسير ردود الطلاب في المرفق (4) ، حصلت مؤشرات الكفاءة على درجة 83.33٪. ويرجع ذلك إلى أن المستخدم أكثر اعتيادًا على الزر الموجود خارج شاشـة هاتفه المحمول بنظام أندرويد، فإن الزر المستخدم إذا تم استخدامه فسيخرج التطبيق تلقائيًا نظرًا لأنه لا يمكن توصيل الزر المقدم من هاتف أندرويد بمعجم وسائط قائم على نظام أندرويد. على مؤشر سهولة الاستخدام يتم تضمين درجة 86.11٪ في الفئة الممتازة. على مؤشر فعالية وسائل الإعلام يتم تضمين درجة 89.35 ٪ في الفئة الممتازة. هذه النتيجة هي أعلى نسبة من جميع مؤشرات تجربة المنتج. استناداً إلى تفسير ردود الطلاب في المرفق (4) ، حصلت مؤشرات الكفاءة على درجة 83.33٪. ويرجع ذلك إلى أن المستخدم أكثر اعتيادًا على الزر الموجود خارج شـاشة هاتفه المحمول بنظام أندرويد، فإن الزر المستخدم إذا تم استخداماه فسيخرج التطبيق تلقائيًا نظرًا لأنها لا يمكن توصيل الزر المقدم من هاتف أندرويد بمعجم وسـائط قائم على نظام أندرويد. على مؤشر سهولة الاستخدام يتهم تضمين درجة 86.11٪ في الفئة الممتازة. على مؤشر فعالية وسائل الإعلام يتم تضمين درجة 89.35 ٪ في الفئة الممتازة. هذه النتيجة هي أعلى نسبة من جميع مؤشرات تجربة المنتج. 
على مؤشر سهولة الاستخدام ، يتم تضمين درجة 85.42 ٪ في الفئة الممتازة ، تحتل هذه الدرجة أعلى ذسبة من جميع مؤشرات تجربة المنتج. وذلك لأن اكتمال الزر ومفتاح الجمع سهل الاستخدام. بالإضيافة إلى مؤشر فعالية وسائل الإعلام الحصيول على درجة 84.9 في المائة في فئلة جيدة جداً ، تحتل هذه المرتبة مركز ثاني أعلى نسبة من درجة مؤشر سهولة الاستخدام. في مؤشرات عرض الوسائط وفوائد الحصول على نفس النتيجة ، أي 83.34٪ ضمن الفئة الممتازة ، تكون هذه الدرجة أيضًا في المرتبة الثالثة من حيث النسبة المئوية بعد درجة مؤشر سهولة الاستخدام. علاوة على ذلك ، حصلت مؤشرات الكفاءة على نسبة 83.33٪ مشمولة في الفئة الممتازة ، وكانت هذه النتيجة مختلفة قليلاً بنسبة 1\% عن مؤشرات ومزايا عرض الوسائط ، ولكنها كانت في أدنى نقطة مئوية لكل مؤشرات تجربة المنتج.

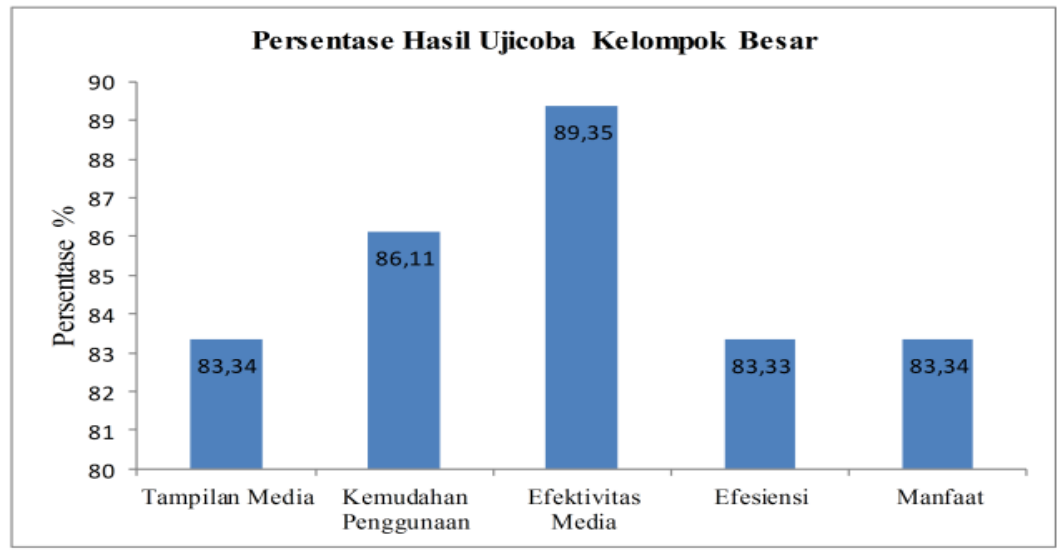

صورة 4.4 النسبة المئوية لنتائج التجربة الجماعية الكبيرة

\section{国 \\ استنتاج}

معجم البيولوجيا القائم على أندرويد على مواد اللافقاريات للطلاب مدرسة الثانوية الصف العاشر ببرنامج الرياضية والعلوم الطبيعية تطورت خلال عدة مراحل بما في ذلك تحليل الصعوبات والأسباب التي يواجهها الطلاب تجاه المواد اللافقارية ، ثم جمع المفردات في مادة اللافقاريات ليتم إدخالها في المعجم ، مما يجعل تصميم محتويات المعجم حتى جذب المستخدمين، ثم تقوم وسائل الإعلام المتقدمة بإجراء التحقق من الصحة بواسطة فريق من الخبراء ، وتنقيح المنتج وفقًا لنصيحة فريق الخبراء ويتم اختباره على طلاب مجموعات صغيرة ومجموعات كبيرة. 
تطوير المعجم ، حصلت على النتيجة النهائية من فريق الخبراء ، أي خبراء المواد أجرى التحقق من صحة 2 مرات وبعد عقد المراجعة على درجة 42 مع نسبة 87.50 \% في معايير جيدة للغاية. علاوة على ذلك ، تم التحقق من صحة خبراء الإعلام ثلاث مرات ، وبعد إجراء التنقيحات قاموا بتسجيل 45 بنسبة مئويـة من 93.75\% في معايير جيدة جـدا. من خلال هذه الاستجابة الجيدة للغاية ، يمكن استخدام وسائل الإعلام واختبارها على طلاب ببرنامج الرياضية والعلوم الطبيعية في المدارس الثانوية من الصف العاشر الذين يدرسون مادة اللافقاريات.

ثم يتم اختبار المنتجات التي تم التحقق منها ومراجعتها للطلاب مدرسة الثانوية الحكومية الصف العاشر ببرنامج الرياضية والعلوم الطبيعياة. استنادًا إلى نتائج تجربة مجموعاة صغيرة على3 طلاب ببرنامج الرياضية والعلوم الطبيعية في الصف العاشر، سجلت المدرسة الثانوية 457 بنسبة 84.01٪ في معايير جيدة جدًا. ثم المضي قدما في تجربة مجموعة كبيرة على الطلاب ببرنامج الرياضية والعلوم الطبيعية 3،2، 1 و 4 درجات X12 فئة المدرسة الثانوية بنسبة 85،55 ٪ في معايير جيدة جدا. من خلال استجابة الطلاب الممتازة ، فإن معجم البيولوجيا القائم على أندرويد على المواد اللافقارية للطلاب من مدرسـة الثانوية الحكومية الصف العاشر ببرنامج الرياضية والعلوم الطبيعية يكون مجديًا لاستخدامه كوسط للتعلم في موضوعات البيولوجيا. اقتراح للاستخدام الأول: يمكن الاستمرار في استخدام المنتجات كمعجم بيولوجي قائم على Android وقادر على استخدامه أكثر تطوراً ، مثل القدرة على البحث عن المفردات باستخدام الصوت والقدرة على وضع جمل إملائية تلقائيًا عند عدم العثور على المفردات.

والثاني: يمكن تطوير قواميس البيولوجيا القائمة على أندرويد في المواد اللافقارية على شكل قواميس ذات قواعد مختلفة مثل القواميس التي تعتمد على (IOS) والمستخدمة على الهواتف المحمولة التي تحمل علامة (Apple) والقواميس التي تعتمد على (Java) والمستخدمة في هواتف نوكيا ، وسوني ، وغيرها من الهواتف المحمولة بحيث يمكن استخدام هذا المعجم على جميع أنواع الهواتف المحمولة ، سواء الإصدارات القديمة والجديدة. والثالث: يمكن إنشاء وتطوير معجم البيولوجيا القائم على أندرويد على المواد اللافقارية للطلاب من مدرسة الثانوية الحكومية الصف العاشر ببرنامج الرياضية والعلوم الطبيعية للطلاب أيضا ، لأن المواد المقدمة في العمق. 
Arikunto, S. (2013). Prosedur Penelitian. Jakarta: Rineka Cipta.

Asim. (2001). Sistematika penelitian pengembangan. Malang: Lembaga Penelitian UNM.

Lee, W. M. \& Owen, D.L. (2004). Multimedia Based Instructional Design. San Francisco: Pfeiffer

Mayer, E, R. (2001). Multimedia Learning. New York : Cambridge University.

Pribadi, B.A. (2009). Model Desain Sistem Pembelajaran. Jakarta: PT Dian Rakyat.

Riduwan. (2012). Belajar Mudah untuk Guru, Karyawan, Peneliti Pemula. Bandung: Alfabeta.

Salbino, S. (2014). Buku Pintar Gadget Android untuk Pemula. Jakarta: Kunci Komunikasi.

Serambimata. (2016). Kurikulum 2013 Edisi Revisi 2016. http://serambimata.

com/ 2016/03/23/inilah-empat-poin-penting-pada-kurikulum-2013-edisi-revisi-tahun2016/. Diakses tanggal 26 Maret 2018

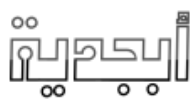

